Alter

Revue de phénoménologie

25 | 2017

L'Histoire

\title{
Polemos est père de toutes choses
}

Guerre et histoire chez Patočka

Bruce Bégout

\section{CpenEdition}

Journals

Édition électronique

URL : http://journals.openedition.org/alter/461

DOI : $10.4000 /$ alter.461

ISSN : 2558-7927

Éditeur :

Association ALTER, Archives Husserl (CNRS-UMR 8547)

Édition imprimée

Date de publication : 3 décembre 2017

Pagination : 193-212

ISBN : 978-2-9550449-3-3

ISSN : 1249-8947

Référence électronique

Bruce Bégout, «Polemos est père de toutes choses », Alter [En ligne], 25 | 2017, mis en ligne le 01 décembre 2018, consulté le 01 mai 2019. URL : http://journals.openedition.org/alter/461 ; DOI :

10.4000/alter.461

Revue Alter 


\title{
POLEMOS EST PÈRE DE TOUTES CHOSES GUERRE ET HISTOIRE CHEZ PATOČKA
}

\author{
Bruce Bégout \\ «J'apporte la guerre» \\ F. Nietzsche
}

Depuis Hegel, la philosophie a lentement mais inexorablement procédé à une historicisation complète de ses concepts. Abandonnant l'éther de la généralité, elle a associé ses notions fondamentales aux événements. Cette historicisation des concepts philosophiques a clairement déplacé le débat du champ de la pensée pure à celui de l'époque, de ses intérêts et de ses conflits. Aussi assistons-nous à une nouvelle gigantomachie qui ne porte plus sur le concept d'être, mais sur les concepts d'histoire eux-mêmes qui ont avalé tout cru l'être et l'ont patiemment digéré comme circonstances, conditions, situations. Ce qui a lieu, depuis deux siècles, c'est l'affrontement violent entre ces concepts d'histoire qui prétendent tous dépasser la dualité du réel et de sa conceptualisation, au profit d'une immanence du sens incarné dans le devenir lui-même. L'esprit du temps a rejeté l'indépendance du réel au profit de sa production. Le concept ne clarifie plus le cours des événements ; il l'engendre directement, non en tant qu'Idée, mais en tant qu'Idée-Réel, idée matérialisée dans la chair du monde, à savoir l'histoire elle-même. L'historicisation de l'être comme technique, capital, vitesse, spectacle, simulacre, etc., accomplit cette dissolution irréversible du processus et du récit.

Pour un grand nombre de penseurs du XXe siècle, le sens de l'époque consiste dans la guerre. Elle en est le chiffre secret, la structure profonde. Une vérité s'impose peu à peu à eux : l'histoire de l'Être s'est accomplie dès l'aube de la modernité comme guerre, une guerre totale, mondiale, perpétuelle. L'ère de la technique elle-même, d'une 
technique sans limites et déchaînée, livrée au déploiement autonome de sa puissance, a accouché de la guerre comme de son événement fondamental. Cela ne signifie pas seulement que la guerre, avec sa cohorte de drames humains et de chaos sociaux, a constitué le fait majeur du siècle passé, et que ses conséquences négatives se font encore sentir en temps de paix, mais qu'elle est devenue le principe de toute réalité historique, à savoir de l'Être devenu histoire. Tous les aspects principaux de la technique - son dispositif d'accumulation et de stockage, son efficacité quantitative niant toute qualité intrinsèque aux choses et aux êtres, son indépendance fonctionnelle formant un monde clos et tyrannique - apparaissent dans et par la guerre mondiale et continuelle. Nous vivons donc à l'époque de la guerre comme horizon unique et infini de l'expérience.

\section{Esquisse d'une phénoménologie de la guerre}

Nul mieux que Jan Patočka n'a su exprimer cette mutation historique de l'Être comme guerre. Le philosophe tchèque a continuellement interrogé ce phénomène spectaculaire et bouleversant. Et ce avant même de lui consacrer une étude spécifique "Les guerres du $X X^{e}$ siècle et le $X X^{e}$ siècle en tant que guerre » ${ }^{1}$. Dès les années trente, il réfléchit déjà à cette césure du monde de la vie qui oblige les hommes à modifier leur mode d'être et de pensée. Il faut dire qu'il aurait fallu être singulièrement détaché de la vie réelle, et perdu dans son monde privé comme le Kien de l'Autodafé de Canetti, pour échapper à ce "phénomène fondamental du XXe siècle ", à "son contenu propre $»^{2}$. On le pressent d'emblée, Patočka ne se contente pas, à la manière d'un Thucydide ou d'un Machiavel, de tirer de la guerre un enseignement philosophique, moral ou politique, il y découvre plutôt « une plénitude de sens difficile à formuler » ${ }^{3}$. La guerre n'est pas tant pour lui un fait, si considérable soit-il, que le fait transcendantal, la manière primordiale dont, à l'époque moderne, l'étant se révèle. Elle forme le mode de manifestation de tout ce qui est, à savoir de tout ce qui est produit par la technique et rendu visible par elle.

$C^{\prime}$ est donc dans un premier temps à partir d'une perspective heideggérienne que Patočka aborde le phénomène de la guerre. Sa ligne interprétative, il la tire de l'analyse de la modernité faite par le philo-

\footnotetext{
${ }^{1}$ J. Patočka, Essais hérétiques (abrégé EH), Lagrasse, Verdier, 1999.

2 EH, p. 154.

${ }^{3}$ Ibid., p. 161.
} 
sophe allemand comme domination planétaire du dispositif technique. La genèse et l'issue des conflits obéissent tous « à l'esprit d'une domination technique sur le monde $»^{4}$. C'est dans la guerre qu'éclate au grand jour le péril du Gestell qui soumet tout ce qui existe et le réduit à quelque chose de disponible, calculable et manipulable. Car, de fait, la technique n'est pas une modification de ce qui est, par exemple une transformation de la nature pré- et anti-technique, mais la production et la manifestation de l'être même. En effet Patočka conçoit la guerre comme le principe d'une mutation de la conceptualité du réel. La guerre bouleverse l'Être, parce qu'elle bouleverse d'abord la manière dont on y accède, par les sens et la pensée. Ce qui, selon lui, différencie en effet la guerre du $\mathrm{XX}$ e siècle, à savoir la guerre techno-scientifique, la guerre comme mobilisation totale des savoirs et des savoir-faire, ce n'est pas le rôle qu'y jouent les idées ; tous les conflits sont idéologiques et mettent en jeu des visions du monde qui s'opposent. Non, ce qui est décisif dans la guerre moderne, c'est le triomphe, par-delà les deux camps réunis dans une croyance commune, d'une même conception idéaliste-pragmatiste de la réalité. Elle représente la "victoire définitive de la conception de l'étant née au XVII ${ }^{e}$ siècle avec l'émergence des sciences mécaniques de la nature et la suppression de toutes les conventions susceptibles de s'opposer à cette libération de forces, une transmutation de toutes les valeurs sous le signe de la force $»^{5}$. Avec la guerre moderne, ce ne sont plus, derrière les hommes, ou plutôt devant eux, les idées qui s'affrontent, mais une seule et même conviction - partagée par tous et par cela même productrice d'un conflit interne, ouvert et permanent - que le réel n'est plus que le résultat d'une décision. C'est cette volonté onturgique qui décide de ce qui est ou n'est pas, de ce qui doit être ou ne doit plus être, qui dirige les opérations. Ce volontarisme technique sape profondément les assisses du réel. N'existe que ce qui est produit. La destruction elle-même ne témoigne pas de la préexistence de l'être sur la technique, mais, à rebours, de sa propre intervention technique comme néantisation; elle est en fait elle-même une production, la production de ce qui n'est pas. Même dans l'annihilation, gouverne donc la loi auto-affirmative de la production technique. La production produit l'être et le non-être ; la destruction n'est pas le contraire de la production, mais une forme particulière de production comme

\footnotetext{
${ }^{4}$ J. Patočka, Liberté et sacrifice, écrits politiques (abrégé LS), Grenoble, Jérôme Millon, 1990, p. 272. En 1973, Patočka a consacré un séminaire privé à l'ère de la technique et aux thèses de Heidegger sur le Gestell planétaire et totalitaire.

${ }^{5} \mathrm{EH}, \mathrm{p} .159-60$.
} 
production de néant, de ruines, de morts. C'est la raison pour laquelle, pour Patočka, la caste militaire prussienne - conservatrice et disciplinée - devient elle-même le bras armé d'une révolution permanente du réel, la «transformation énergétique du monde $»^{6}$. Tout ce qu'elle peut - techniquement - accomplir, elle a le devoir de l'accomplir. Aux yeux du philosophe praguois, il ne suffit pas de penser, comme une région eidétique particulière qui aurait ses lois et ses manières, une ontologie de la guerre, à savoir une polémologie ; il est nécessaire de concevoir de manière plus fondamentale une ontologie en guerre, où la nouvelle "guerre des Dieux» qu'évoquait Weber dans Le savant et le politique ${ }^{7}$, ne concerne plus les valeurs, mais prend place au sein même de l'Être parce qu'elle est cet Être même. C'est le concept d'Être lui-même qui est bouleversé de fond en comble par " cette époque de nuit, de guerre et de mort ${ }^{8}$.

Mais que veut cette volonté de puissance technique qui fait de la guerre le moyen de son accomplissement mondial ? Rien d'autre, selon Patočka, que l'accumulation de sa propre puissance. Elle n'est pas accumulation de capital, mais de forces. Grâce aux appareils et aux méthodes, elle produit d'énormes stocks de puissance: forces matérielles et humaines, réservoirs d'énergie et masses mobilisables. Or cette accumulation gigantesque, qui naît avec la révolution technoindustrielle et modifie le paysage terrestre, afin de ne pas être ellemême détruite par son trop-plein, exige de temps en temps une décharge violente:

La grande guerre est l'événement décisif de l'histoire du $\mathrm{XX}$ e siècle. C'est elle qui décide de son caractère général, qui montre que la transformation du monde en un laboratoire actualisant les réserves d'énergie accumulées pendant des milliards d'années doit se faire par voie de guerre?

La guerre apparaît alors pour Patočka, paradoxalement, comme «le moyen le plus efficace de libérer rapidement les forces accumulées $\gg^{10}$. C'est, pour être précis, le moment de l'autorégulation de la puissance qui se déchaîne pour ne pas se mettre en péril. Autrement dit, la guerre appartient à la surcivilisation ${ }^{11}$ qui fait de la décharge

\footnotetext{
${ }^{6}$ Ibid., p. 160.

${ }^{7}$ M. Weber, Le savant et la politique, trad. C. Colliot-Thélène, Paris, La découverte, 2003, p. 103.

${ }^{8} \mathrm{EH}, \mathrm{p} .155$.

${ }_{9}$ Ibid., p. 159.

${ }^{10} \mathrm{EH}$, p. 160.

${ }^{11}$ Sur le concept de surcivilisation, cf. «La surcivilisation et son conflit interne », in LS, p. 99-180, et « La civilisation technique est-elle une civilisation en déclin et pourquoi ?, in EH, p. 125-152. Et nos propres commentaires, in Le phénomène et son ombre, Chatou, La Transparence, 2007.
} 
orgiaque et démonique le cœur de son développement rationnel. Cette décharge a lieu en temps de paix dans le divertissement de masse, le spectacle consolant et abrutissant, mais elle se produit aussi, et selon la même logique, au cours de la guerre mondiale, cette « libération de potentialités orgiaques qui nulle part ailleurs ne peuvent se permettre de porter l'ivresse de la destruction jusqu'à cette dernière extrémité $\gg^{12}$. Comme le divertissement, la guerre offre la possibilité d'un potlatch d'énergie sans destination :

$\mathrm{Au} X X^{\mathrm{e}}$ siècle, la guerre, c'est la révolution de la quotidienneté d'ores et déjà accomplie. Révolution qui s'accompagne d'un mouvement de libération et d'un happening universels, d'une recrudescence de l'orgiasme sous des formes nouvelles ${ }^{13}$.

Un même enthousiasme chemine devant les vitrines et sur le champ de bataille. À l'instar du travail et des loisirs, la guerre représente « la plus grande entreprise de la civilisation industrielle $»^{14}$. Sauf que le travail et le loisir ne suffisent pas manifestement à épuiser - et réguler - les forces colossales que l'époque de la technique a libérées et stockées. Ils laissent une quantité de forces sans emploi, ce qui se traduit chez ceux qui les ont accumulées, et qui en font l'expérience quotidienne, par une frustration et une nervosité exacerbées; la guerre intervient alors comme l'acte par lequel la surpuissance s'auto-consume - partiellement - afin de se sauver. Ce qui ressort de ces remarques, c'est que l'orgiasme industriel de la surcivilisation se donne comme le nouvel empire, la totalité protectrice qui décide par avance du sens et de la vie, empire qui orchestre la production/destruction de l'étant dans le monde des loisirs de masse comme dans la fureur guerrière. Une même planification de « la science technicisée » ${ }^{15}$ détermine l'organisation du temps de paix entre travail et loisir et la libération violente de la force pendant la durée de la guerre.

Cependant la guerre ne représente pas seulement cette expression terrible de la domination du Dispositif par le biais de la mort violente. Peu à peu, Patočka se dégage de ce schéma heideggérien dont il conteste les applications pratiques (notamment le laisser-être serein et quelque peu passif face à la catastrophe) comme certains présupposés théoriques. A partir de Heidegger, mais au-delà de lui, il fait rapidement entendre un ton plus personnel. Le phénomène de la guerre $\mathrm{s}$ 'inscrit alors dans une réflexion générale sur le sens de l'existence

\footnotetext{
12 EH, p. 146.

${ }^{13}$ Idem.

${ }^{14}$ Idem.

15 Idem.
} 
humaine et de l'histoire, sur le "souci de l'âme». Dans ses conséquences ultimes et désastreuses, il s'inscrit dans ce que Patočka nomme la «logique du jour». Qu'entend-il par là? Le jour, c'est pour ainsi dire la puissance de la raison qui détermine de fond en comble l'organisation sociale comme idéologie du bon sens. C'est l'élimination de l'obscurité, à savoir de tout ce qui résiste à la formulation rationnelle, de tout ce qui ne peut pas se montrer comme étant manifeste, compréhensible et calculable. Le jour désigne donc l'empire de l'évidence et le refus de la problématicité. C'est l'autre nom de la rationalité instrumentale qui se met au service de la vie biologique, conçue préalablement comme immanence pure, reproduction sans rupture ni transcendance.

En quoi la guerre mondiale est-elle soumise aux forces du jour? Pour le comprendre, il faut tout d'abord se rendre compte que, pour Patočka, c'est le jour lui-même comme puissance rationnelle et technique attachée à la vie qui mène continuellement la guerre. Celle-ci n'est pour lui que le moyen d'assoir un peu plus sa domination. L'optique du jour, c'est l'attachement à la vie réduite à la perpétuation biologique, à sa planification et à son entretien. Il n'est donc pas étonnant que, "dans la volonté de guerre, ce sont donc le jour et la vie qui règnent par le moyen de la mort ${ }^{16}$. Même la paix est la continuation de cette hégémonie impériale du jour. La guerre continue dans la paix, par la paix, car elle est l'expression de la même conception de l'Être comme force et calcul. Avec la paix, la guerre a changé insidieusement de forme "sans prendre fin $»^{17}$. Il n'y a pas là de paradoxe ni de provocation. La paix est bien l'œuvre du jour qui vise la conservation de la vie. Cependant la guerre militaire, géopolitique, technologique et économique entre puissances se poursuit aussi en temps de paix :

Or ce qui triomphe dans ce combat brutal, c'est derechef la Force qui emploie la paix comme un moyen de combat, si bien que la paix devient partie intégrante de la guerre, cette étape artificieuse de la guerre qui écrase l'ennemi sans coup férir - du fait qu'il ralentit la mobilisation $[\ldots]^{18}$.

À ces forces du jour, «il leur est égal d'organiser ou de détruire ${ }^{19}$. L'objectif de guerre est toujours un objectif de paix. En d'autres termes, le jour fait la guerre en vue de la paix, de l'ordre qu'il par-

\footnotetext{
${ }^{16}$ Ibid., p. 165.

17 Ibid., p. 169.

${ }_{18}$ Ibid., p. 163.

19 Ibid., p. 160.
} 
viendra à imposer : «La perspective de la paix, de la vie et du jour est sans fin, c'est la perspective d'un conflit interminable, qui renaît sans cesse, identique sous des figures toujours nouvelles $»^{20}$.

Aussi la paix fait-elle "partie intégrante de la guerre ». Et la guerre elle-même n'est qu'une " passation de fonctions » ${ }^{21}$ et " s'installe durablement par des moyens pacifiques $»^{22}$. En effet les motifs de la guerre demeurent diurnes, $c^{\prime}$ est-à-dire qu'ils obéissent entièrement à l'espoir d'une défense et d'une augmentation de la vie. Toute guerre se déclenche toujours en fonction de la vie qu'il faut protéger, accroître, glorifier. C'est sa face «pacifique ${ }^{23}$. Ce sont ces mêmes motifs qui gouvernent la vie quotidienne en temps de paix et expriment la logique pure du jour. Et ce sont eux qui servent de fins ultimes au soldat : «La paix et le jour ne peuvent régner qu'en envoyant des hommes à la mort afin d'assurer à d'autres un jour à venir sous les espèces du progrès, d'un développement lent et continu ${ }^{24}$. L'attachement à la vie qui mobilise la force et la raison conduit naturellement à la guerre, à une guerre permanente et sans fin. Dans la guerre même, l'homme est encore enchaîné à la vie par la mort et par la peur, et devient donc ainsi soumis «manipulable à l'extrême $»^{25}$.

Cependant la guerre n'est pas que l'affirmation de la vie dans la mort. En elle, il y a quelque chose qui conteste "l'optique du jour », quelque chose qui échappe à la logique de l'auto-conservation immanente du vivant. Si la guerre est habituellement le moyen pour la paix $\mathrm{du}$ jour de s'imposer, elle laisse aussi percer de manière marginale mais pour autant essentielle une expérience qui échappe à cette mainmise, une autre guerre dans la guerre. Il ne faut plus alors considérer la guerre dans la seule perspective du jour, mais, à rebours, par son " côté ténébreux, sombre ${ }^{26}$. Cet autre aspect de la guerre qui n'entre plus dans les calculs de la raison visant à conserver et augmenter la vie, parfois au prix de la destruction elle-même, c'est l'expérience du front qui le révèle. Patočka voit dans cette expérience - notamment

\footnotetext{
${ }^{20}$ Ibid., p. 169.

${ }^{21}$ Ibid., p. 154.

22 Ibid., p. 170.

23 Idem.

${ }^{24}$ Ibid., p. 165.

${ }^{25}$ Ibid., p. 170. Cf. également, p. 166 : «La paix, transformée en volonté de guerre, réussit à faire de l'homme une chose purement extérieure aussi longtemps que l'homme est régi par le jour, par l'espoir d'un quotidien, d'une profession, d'une carrière, bref, de possibilités pour lesquelles il se voit obligé de craindre et qu'il sent menacées ».

${ }^{26} \mathrm{Ibid}$., p. 154. Cf. également, p. 170 : «Il semble y avoir aussi, pour cette raison même, une possibilité de sortir de la guerre engendrée par la paix, de reprendre pied dans une paix réelle. Cela présuppose en premier lieu l'expérience du front $[\ldots]$ ».
} 
celle de la Première Guerre mondiale - une espèce de libération existentielle, un affranchissement de la vie, un ébranlement du sens donné. Cette interprétation valorisante $\mathrm{du}$ front, cette reconnaissance philosophique de la " positivité de la guerre», est pour le moins étonnante, voire choquante; $c^{\prime}$ est une "idée hérétique » ${ }^{27}$, et elle représente sans doute la plus grande hérésie que contiennent les Essais hérétiques.

\section{La polémique du jour et de la nuit}

Prenant le contrepied du pacifisme moral qui constitue bien souvent la position philosophique dominante, libérale et humaniste, Patočka va avancer par petites touches un bellicisme assumé : «La grande expérience du front avec sa ligne de feu consiste cependant à évoquer la nuit en tant que présence impérieuse qui ne peut être négligée $»^{28}$. Mais qu'y a-t-il de si positif et exaltant dans l'expérience de la guerre? N'est-elle pas le déchaînement nihiliste de la technique ? L'annihilation catastrophique et méprisable de la vie ? Assurément, mais elle est aussi, et de manière plus souterraine, la contestation radicale de la logique du jour. La découverte la plus fondamentale que permet l'épreuve du front, c'est, selon Patočka, le détachement soudain et abyssal qu'il provoque vis-à-vis de la défense de la vie, des forces du jour. Cette position audacieuse ne témoigne pas, comme on pourrait le croire dans une lecture trop rapide, d'un nihilisme jouissif de la destruction ni d'un romantisme noir et provocateur, mais elle renvoie à une expérience philosophique authentique, celle de «l'ébranlement du jour $»^{29}$, de la paix comprise comme domination sociale de la vie et de l'ordre. Cette guerre dans la guerre qu'essaie de penser Patočka est aussi une guerre pour la guerre, une guerre qui ne se déroule pas en vue de la paix, de l'ordre, de la vie réduite à la conservation biologique et au sens donné, mais continuellement contre eux. Là où le fasciste fait de la guerre le principe universel et éternel de la vie ${ }^{30}$, Patočka les oppose. En fait, il objecte à la guerre de la vie et du jour, le combat spirituel de la nuit. Par

\footnotetext{
27 Idem.

${ }^{28}$ Ibid., p. 165.

${ }^{29}$ Ibid., p. 167.

${ }^{30}$ Voici un extrait d'une publication nazie des années trente, le SS-Leitheft : «Force contre force, voilà le caractère éternel de la vie [...]. Dans la nature, les forces se combattent sans cesse les unes les autres. La mer se jette sans relâche contre la falaise que la terre a édifiée pour se protéger d'elle, la tempête attaque sans discontinuer la forêt pour briser les arbres [...]. La guerre éternelle est la loi de la vie ", cité dans J. Chapoulot, La loi du sang. Penser et agir en nazi, Paris, Gallimard, 2014, p. 204.
} 
conséquent, cette autre guerre est l'épreuve du détachement de la vie immanente, une sorte d'ascèse, de renoncement brutal au confort vital et social, l'accès à quelque chose d'autre, de plus problématique, de plus secret. Le conflit dans l'existence n'est pas le «struggle for life » ou le «Alles Leben ist Kampf ». Au contraire il s'écarte de toute (pseudo-)loi naturelle pour se poser comme rupture avec le monde de la vie. Si la guerre du jour, qui a lieu aussi en temps de paix en tant qu'industrie culturelle et libération de «potentialités orgiaques ${ }^{31}$, bouleverse continuellement l'homme et sa vie, elle ne les ébranle pas profondément. L'ébranlement ici en question n'est pas l'expression de la mobilité permanente du monde technicisée, sa vitesse et sa fureur, ni même celle de la lutte sociale et de la concurrence capitaliste dans l'optique du darwinisme social, il implique une rupture totale avec cette logique orgiaque. Patočka oppose très clairement au bouleversement de la surcivilisation (la « révolution de la quotidienneté » ${ }^{32}$ ) qui mobilise jour et nuit les corps et les esprits et les soumet sans reste au diktat de la raison instrumentale, l'ébranlement politique et philosophique qui ne met pas sens dessus dessous l'étant, mais le congédie bel et bien. C'est de cet ébranlement-là qu'il s'agit dans l'autre guerre.

$\mathrm{Au}$ cœur de l'expérience du front se trouve donc « une positivité profonde et mystérieuse $»^{33}$. C'est comme si la guerre provoquait une conversion de l'existence à la recherche d'un sens plus haut, plus vaste, plus complexe que le simple sens donné par l'ordre vital et social qui domine le jour. La positivité de la guerre réside ainsi pour Patočka dans cette opposition aux valeurs du jour. Certes le front, tel que peuvent le décrire les rescapés, enfin ceux qui osent encore parler et ne se réfugient pas dans le mutisme, apparaît au premier abord dans la furie destructrice et l'effroi total comme l'expérience même du non-sens. Demeure pourtant en lui, juge Patočka, «quelque chose comme la fin de toutes les valeurs du jour » : «Ce front, c'est celui de la résistance aux motifs démoralisants, terrorisants et trompeurs du jour $»^{34}$. Le front nous affranchit de la servitude du jour. Afin de comprendre cette positivité scandaleuse de la guerre, il convient de faire retour sur le sens de la puissance du jour.

La critique de la paix par la guerre ne prend sens qu'à partir de l'analyse du monde de la vie quotidienne. Dès son travail de thèse, Patočka a soutenu l'idée que le monde naturel se caractérise par un

\footnotetext{
${ }^{31} \mathrm{EH}$, p. 147.

${ }^{32}$ Ibid., p. 146.

${ }^{33}$ Ibid., p. 161.

34 Ibid., p. 171.
} 
certain rapport au sens. Le monde de la vie est avant tout celui de la "vie nue », à savoir de la vie réduite à sa simple perpétuation, à sa défense et à sa croissance, bref à son déploiement immanent. Cette conservation ne concerne pas uniquement l'individu biologique, mais la société tout entière et son organisation. Le monde naturel et préhistorique se présente donc comme le monde du « petit rythme de la vie », du sens modeste mais sûr. Ce monde pré-historique ne connaît pas encore la césure brutale que vont introduire la philosophie et la politique comme critiques de la tradition du sens donné et recherche infinie d'un sens plus riche et élevé. Les grands empires pré-historiques reposent eux-mêmes tout entier sur le sens donné et garanti : par l'État, la religion, la tradition. Ces structures forment des totalités closes dans lesquelles le sens de la vie est toujours fini et défini. Au fond, ce sont, en dépit de leur aspect grandiose, de simples entreprises de gestion quotidienne. Elles ont le même but que la vie individuelle prise dans ses petits efforts ordinaires de perpétuation. Elles s'apparentent ainsi à des organisations étatiques de l'oikos, reposant sur une économie domestique étendue à l'échelle vaste d'un empire. Mais, entre elles et la vie quotidienne banale, il n'y a qu'une différence de degré, non de nature. Un même principe les régit : « Les premiers empires donnent à l'existence humaine le même sens que connaît déjà l'homme : celui de pourvoir à la subsistance commune ${ }^{35}$.

Or c'est ce principe immanent, autotélique et aveugle que la philosophie et la politique mettent en question. Il serait trop long ici de restituer cette analyse complexe de la formation du monde historique par la césure critique. Contentons-nous de retenir seulement ce qui renvoie à la logique du jour. Dans le monde de la vie préhistorique, donc de la vie nue (réduite à sa reproduction totale) et traditionnelle, c'est le jour qui organise et régit le sens. Cette logique est contestée par l'irruption grecque de la problématicité, c'est-à-dire de la critique et de la recherche, de l'attitude que les anciens nommaient zététique, mais elle se reforme - et se referme - étrangement aux temps modernes avec la puissance de la technique et de la raison instrumentale. Dans la surcivilisation, c'est la logique du jour nonhistorique et biologique qui domine de nouveau. Le système technoscientifique a remplacé l'ordre mythologique du sens total et incontestable, mais il impose de la même manière un refus du questionnement et une évidence commune qui organisent la vie dans le travail, les loisirs et la guerre impérialiste. La rationalité a pris le relais des dieux et détermine en totalité le réel.

35 Ibid., p. 59. 
L'histoire ne commence véritablement que lorsque ce monde de la vie quotidienne, qu'il soit fondé sur des assises mythologiques ou technologiques, prémodernes ou hypermodernes, est remis en cause par une critique radicale de son sens. Ce n'est pas tant ce sens donné qui y est d'ailleurs contesté, questionné, amendé que le rapport même des hommes au sens. L'historicité ne débute pas par l'écriture, mais par l'ouverture à un horizon de sens non donné, à savoir par un refus des évidences. Tel est le sens que Patočka confère au terme d'ébranlement ${ }^{36}$. Le conflit est l'expérience-source de l'historicité humaine. L'homme ne vit pas seulement dans l'histoire, mais il vit véritablement l'histoire, lorsqu'il est capable de remettre en cause, jusqu'à la critique la plus radicale, le sens donné par la vie et la tradition qui n'en est que la forme sociale. L'ébranlement, c'est à la fois cette perturbation du sens donné dans le monde de la vie pré-historique et la quête d'un sens plus ample et plus ambitieux. Or l'ébranlement va de pair avec la problématicité, il en est l'effet immédiat. En un sens, le monde de la vie déproblématise immédiatement le rapport de l'homme au monde et à son existence en fournissant des réponses toutes faites, de systèmes de sens clés en main : la mythologie religieuse et l'idéologie de l'État. Ce monde naturel a une très grande réponsabilité, une capacité de donner des réponses à tout qui refoule le caractère problématique et ouvert de l'existence. L'avènement de l'histoire s'opère en revanche lorsque les hommes osent ne plus se satisfaire de ces évidences données et s'engagent dans un combat contre elles, dans un long et pénible cheminement qui est celui de la problématicité ellemême. Contre le dogmatisme du jour, se lève donc l'existence authentique et historiale du philosophe qui vit, non dans les lumières totalisatrices, mais dans un rapport obscur, complexe et profond au sens, dans une atmosphère d'autocritique et de problématicité. Voilà pourquoi l'ébranlement du sens ne ressortit pas au nihilisme de la destruction $^{37}$, mais entre en conflit avec le sens donné. La quête du sens refuse à la fois la vie déjà dotée de sens (la tradition dogmatique) et la vie dénuée de sens (le nihilisme insensé). Ce combat est au centre de ce que Patočka nomme, à partir de 1965, le «troisième mouvement de l'existence humaine ». À la lutte pour la vie qui caractérise les deux premiers mouvements (celui de l'acceptation/adaptation et celui de la

\footnotetext{
${ }^{36}$ LS, op. cit., p. 291 : «Qu'est-ce à dire ébranler ? L'ébranlement montre qu'il y a, dans ce qui se manifeste à moi et m'embrasse en son sein, quelque chose de labile, une solution de continuité, une faille - il apparaît que l'étant qui m'englobe repose sur autre chose qui ne relève pas de ce contexte ».

${ }_{37}$ Patočka reconnaît cependant qu'il existe un ébranlement nihiliste qui détruit sans reste le sens et s'oppose à toute recherche d'un sens plus fort et plus ample, cf. EH, p. 104.
} 
défense/croissance), s'oppose la lutte contre la vie: le mouvement pour la vérité. L'existence elle-même est traversée par cette dialectique entre le jour et la nuit, entre l'adhésion au sens donné et la confrontation à l'Ouvert. Elle cherche à atteindre "une teneur de sens plus libre, plus ambitieuse $»^{38}$. Dans ces conditions, polemos ne désigne plus ici la guerre faite au nom du jour, mais la lutte historiale contre le jour, lutte qui, selon Patočka, définit la philosophie et la politique démocratique en tant qu'elles ébranlent le sens reçu, et promeuvent une nouvelle forme de vie, critique et dialectique. Alors que la vie nue et traditionnelle se dérobe à la problématicité, se réfugiant dans le mythe et l'État, la vita nova s'y expose. Or cette lutte nouvelle et historique repose sur des vertus guerrières : le courage, la détermination, le sens du sacrifice. La guerre permet à l'homme d'affirmer sa supériorité sur la vie, sur sa propre vie. Il montre qu'il est capable de mépriser l'instinct de survie et d'agir en fonction de ce qui ne relève plus de la bio-logique.

\section{Les deux guerres et la véritable paix}

Ce qui se joue dans la guerre ce n'est pas donc pas uniquement la puissance de la vie et son expression productrice/destructrice, mais un combat entre la vie et l'existence. La guerre laisse transparaître un dépassement de la vie dans l'existence, de la clôture biologique et sociale dans l'ouverture. C'est la lutte entre «plus de vie » et «plus que la vie » qui a lieu derrière le déploiement orgiaque de la production/destruction des vies. Il existe donc pour Patočka deux guerres dans la guerre : 1) la guerre faite par la vie, dans la mort et par la mort, qui se prolonge en temps de paix comme rationalisation froide et défoulement orgiaque ; et 2) l'ébranlement courageux, périlleux, nocturne de cette puissance. Comme Hegel, Patočka perçoit dans la guerre un ébranlement, mais ce n'est plus celui de l'égoïsme des familles, de la vie civile et de ses valeurs, au profit de l'Etat comme représentant de l'Universel, mais celui du sens total et clos. La guerre par le sacrifice qu'elle implique montre qu'il existe quelque chose de plus que ce qui est donné. La mort devient le maître de cette révélation.

Relisant dans des «pages étonnantes » Jünger et Teilhard de Chardin, Patočka développe une lecture philosophique du front qui va dans le sens de cet ébranlement problématique du monde de la

38 Ibid., p. 88. 
vie. Il n'hésite pas à voir en effet dans cette épreuve terrible, geyser de feu et de fer, une sorte d'expérience intérieure, une Erlebnis profonde, nocturne, une forme de transfiguration de l'existence qui ne laisse rien en l'état: "La découverte la plus profonde du front, c'est cette avancée de la vie dans la nuit, le combat et la mort [...] ; c'est la transformation du sens de la vie qui heurte ici au néant, à la frontière infranchissable qui est la figure du tout ${ }^{39}$. Avec une certaine grandiloquence, Patočka confère à l'expérience de la guerre un sens philosophique, voire métaphysique. Il dépasse ainsi la vision pessimiste de l'absurdité de la guerre où l'homme ne ressent qu'une "expérience du non-sens et un effroi insoutenable ${ }^{40}$. Il décrit plutôt l'expérience $\mathrm{du}$ front comme une conversion spirituelle, une « metanoia » ${ }^{41}$ de l'âme qui arrache l'homme à la certitude rassurante et non historique du quotidien, qu'organise la puissance du jour. Si la décision philosophique de s'arracher à l'étant, et à la pensée qui le domine, n'appartient qu'à quelques-uns, les héros philosophiques et politiques, la guerre fait office pour le plus grand nombre d'une révélation commune et massive qui convainc, au milieu des cadavres et des cendres, qu'il y a autre chose que cet étant calculable et manipulable, autre chose qu'un " magasin de forces » ${ }^{42}$. Patočka n'hésite donc pas à voir dans la guerre une chance, la possibilité d'un revirement existentiel de l'homme qui, face au néant de la vie et du sens, se détache d'une certaine logique réductrice et totalitaire qui les gouvernait, celle du jour, de la rationalité instrumentale et du Gestell, pour s'ouvrir tragiquement à un horizon problématique, celui de l'historicité elle-même. Mais le prix à payer de cette révélation n'est-il pas trop lourd ? Seule une catastrophe peut-elle nous sauver?

Ce qui est sûr pour Patočka, c'est qu'il existe une positivité du front et de la négativité, le « front non pas comme asservissement à la vie, mais comme libération infinie et affranchissement de cette servitude $»^{43}$. Le philosophe tchèque $n^{\prime}$ en minimise pas les souffrances et

\footnotetext{
${ }^{39}$ Ibid., p. 167.

${ }^{40}$ Ibid., p. 161.

${ }^{41} \mathrm{Ibid}$., p. 172. C'est la même expression que Patočka emploie pour décrire le changement de perspective du philosophe qui abandonne le sens donné de la tradition pour le «sens en tant que chemin », cf. EH, p. 102 : « une conversion colossale, un metanoein sans précédent ».

${ }_{42}$ LS, p. 317.

${ }^{43} \mathrm{EH}$, p. 171. On retrouve chez Jünger cette même idée selon laquelle l'expérience de la guerre nous arrache à la chaîne des jours. L'esprit de la guerre révèle l'insatisfaction de la vie quotidienne, l'ouverture au sauvage et à la lutte. Cf. E. Jünger, La guerre comme expérience intérieure, Paris, Bourgois, 1997. Mais l'écrivain allemand essentialise la guerre comme libération des pulsions contenues dans et par la civilisation, comme intensification de la vie et mode d'existence original. Dans cette perspective quasi esthétique, l'ennemi lui-même n'a plus de visage et le conflit de but. Ce qui est essentiel est le type de mobilisation de la vie que procure la guerre,
} 
les horreurs, il en mesure au contraire l'extrême négativité. Mais cette négativité est aussi celle qui nie un certain rapport à l'étant, à la vie et au sens. Seule, pour lui, l'exposition totale et sans défense de l'homme au front, au feu et à la force, peut d'une certaine façon lui permettre de dépasser la logique du jour et accéder à sa propre historicité. Comment? En lui faisant prendre conscience qu'il y a justement quelque chose qui dépasse la vie et qui ouvre à une liberté absolue. C'est dans le péril extrême qu'apparaît le retournement ultime. Dans l'optique du jour, la vie ne peut transcender la vie; elle vise avec une obstination admirable à sa seule conservation et à sa seule croissance, et ce pour l'éternité. Ce sont la mort violente et la destruction de l'étant qui obligent cette vie à une Aufhebung dans l'existence. Comme dans le schéma apocalyptique de Heidegger, le « salut » (das Rettendes) advient à la fin des temps lorsque la puissance du dispositif planétaire s'est déployée jusqu'à ses extrémités dernières et catastrophiques: "En tout cas, il faut aller jusqu'à la dernière extrémité, là où l'évidence du monde technique devient monstruosité, où nous nous apercevons que nous nous trouvons devant un abîme, nihil, que le sol se dérobe sous nos pieds, que nous restons suspendus dans le vide, et c'est là dans ce suspens, qu'on trouvera le joint ${ }^{44}$. Là, uniquement, peut poindre une ouverture, une sortie hors de la calculabilité. La guerre fait aux hommes, contre leur volonté, ce que la philosophie et la politique n'ont pas réussir à provoquer manifestement chez eux : l'arrachement des certitudes ontiques et vitales, du monde de la vie comme sol et horizon rassurants. Là où l'événement de la problématicité ouvre à la fois le règne de la liberté et de l'histoire, le front violent opère cette même brisure, en ne détruisant pas seulement les certitudes, mais les étants qui les supportent. Ce que Patočka désigne par «vie au sommet », c'est cette vie qui, mise au pied du mur, se hausse au-dessus de la vie nue dans l'ouverture au problématique, à la mort du sens. Elle seule accède à l'ampleur, au rejet de la simple organisation bio-politique de la vie qui n'a d'autre fin que la satisfaction autonome et circulaire d'un processus immanent: "La vie est à comprendre, non pas du point de

\footnotetext{
l'augmentation de la volonté de puissance. «La cause n'y fait rien, tout est dans la conviction » (p. 160), c'est-à-dire tout est dans l'engagement vital et existentiel du guerrier. C'est une expérience pure de la volonté et des sens. Là où Jünger fait de la guerre un fondement de l'être comme lutte et puissance, Patočka rejette cette vision naturalisatrice et essentialiste pour percevoir dans la guerre, non le moment de l'intensification, mais celui de la problématisation. La guerre n'est pas la chose en soi du monde, le vouloir-vivre dans son déploiement sauvage et brutal, mais la suspension de l'en soi au profit de la néantisation de tout donné : la vie, l'étant, le sens.

${ }^{44}$ LS, p. 319.
} 
vue du jour, dans la seule optique de la vie acceptée, de la vie pour la vie, mais aussi du point de vue du conflit, de la nuit, du point de vue du polemos $\gg^{45}$.

Cette conversion du sens, du sens donné en sens problématique, repose incontestablement sur un sacrifice. Mais pas n'importe quel sacrifice. Force est de constater que la logique du jour en appelle ellemême continuellement au sacrifice; la destruction qu'entraîne la guerre est précisément justifiée comme un sacrifice nécessaire à la perpétuation du sens et de la vie pris comme totalités. Ce sacrifice du jour est toujours un sacrifice relatif, relatif à ce qui dépasse la guerre et se pose comme paix. Le sacrifice qui est demandé au soldat, comme à toute la société civile, a pour finalité d'assurer à d'autres la vie, le progrès, le confort. Il s'agit donc d'un sacrifice au nom du jour. C'est là, indique Patočka dans une conférence de 1973, un sacrifice "naïf », " un sacrifice au sens objectif, on pourrait presque dire chosique ${ }^{46}$. À l'opposé de ce sacrifice-pour-quelque-chose-ou-quelqu'un, qui n'est en fin de compte $\mathrm{qu}^{\prime}$ « un rendement $»^{47}$, et participe de l'essence de la technique, l'expérience violente et insoutenable du front exige le sacrifice pour rien :

La reprise $\mathrm{du}$ sacrifice présuppose davantage que l'immolation volontaire que représente le sacrifice naï. Dans la répétition, il y va au fond du dépassement de la compréhension technique de l'être, responsable de la méconnaissance et de la vanité des sacrifices. L'attitude naïve ne suffit donc pas, et ce qui se porte au premier plan n'est plus le pour-quoi concret du sacrifice. L'action est, au contraire, à comprendre dans la seule perspective de la protestation, comme un non qui ne s'adresse pas à des états de fait singuliers et concrets, mais au mode de compréhension qui les sous-tend. Vu dans cette optique, le sacrifice repris en est un dont l'enjeu n'est rien d'affirmatif, rien qui ait un contenu positif ${ }^{48}$.

Ce sacrifice, qui est un « se-sacrifier pour ce qui n'est aucune chose ni rien de chosique ${ }^{49}{ }^{9}, n^{\prime}$ a d'autre but que de nier ce qui appartient à la logique du monde et de la vie. Il ne s'effectue pas d'abord en vue d'une ampleur de vue, d'une quête de sens. Il ne marchande pas un gain. Sa vérité consiste dans son acte pur et absolu, «cela veut dire que le sacrifice de ces sacrifiés perd sa signification relative [...], il n'a

\footnotetext{
${ }^{45} \mathrm{EH}$, p. 68.

46 «Les périls de l'orientation de la science vers la technique selon Husserl et l'essence de la technique en tant que péril selon Heidegger », in LS, p. 274.

${ }^{47}$ LS, p. 321 : «Dans le sacrifice authentique, il n'y a rien de semblable, il ne s'agit pas d'un échange, je ne paie pas pour acquérir autre chose. Le sacrifice inauthentique donne un rendement. Ici il n'y a pas de rendement, c'est une non-performance ».

${ }^{48}$ LS, p. 274.

${ }^{49}$ Idem.
} 
de sens qu'en lui-même ${ }^{50}$. Or ce sacrifice pour rien, pour rien de ce qui relève de la vie, du jour et de la paix, ce sacrifice qui paraît absurde et sans raison ( figure singulière et paradoxale $»^{51}$ ), encore plus pitoyable que le déchaînement de violences qui l'entoure, $\mathrm{s}^{\prime}$ avère en fin de compte un renoncement salvateur ${ }^{52}$. Seul ce sacrifice radical peut devenir «le point de départ d'un revirement dans notre compréhension du monde et de la vie $»^{53}$. Si par conséquent «la guerre, c'est la preuve ad oculos que le monde est désormais mûr pour la fin $»^{54}$, cette fin n'est que la fin de toutes choses, mais pas forcément la fin de ce qui n'est pas chose ou conçue comme chose.

Mais, tout en condamnant avec force le caractère instrumental de la guerre techno-scientifique comme moyen de consolidation de l'ordre vital (lequel n'est qu'une "guerre larvée » ${ }^{55}$ ), Patočka ne s'appuie-t-il pas lui aussi sur une certaine conception médiale de la guerre en en faisant le moment révélateur de dépassement de l'étant? Dans la perspective du jour, la guerre sert à obtenir une paix future (la foutaise d'un monde meilleur qui n'est autre que le même régi par le même), dans la perspective de la nuit, à révéler l'au-delà de l'étant et de la force. Elle n'est donc pas tout à fait dans le second cas un sacrifice pour rien. C'est seulement dans la perspective du jour, et donc de l'étant, qu'elle apparaît comme négativité totale. En tant que sacrifice authentique et radical, elle possède une positivité. Il s'agit cependant d'une positivité spéciale qui ne renvoie pas à l'être posé, connu et utilisable. C'est celle de la liberté, d'une liberté qui s'affirme dans la négativité totale et dans le rejet de toute médialités6. La guerre n'est pas un moyen en vue de, de la paix, de la sauvegarde d'une société ou des siens, mais une guerre pour la guerre, guerre absolue qui ouvre la liberté humaine dans le dessaisissement de soi :

Cette liberté absolue, c'est la compréhension que quelque chose là a d'ores et déjà été atteint, quelque chose qui n'est pas un moyen en

\footnotetext{
${ }^{50} \mathrm{EH}, \mathrm{p.} 166$.

${ }^{51}$ LS, p. 275.

${ }^{52}$ Cf. ibid., p. 272 : «Or l'expérience du sacrifice est devenue l'une des expériences prépondérantes de notre époque, expérience tellement forte et déterminante que l'humanité, incapable pour la plupart de s'expliquer avec elle, se réfugie pour y échapper, dans la compréhension technique de l'être ". L'ère technique n'est pas simplement la négation de l'idée même de sacrifice, dans le sens où elle réduit tous les étants à « des fonds utilisables » (p. 272); elle exige aussi, à bien y regarder, un sacrifice, mais c'est un sacrifice utile et raisonnable en vue de quelque chose, donc un sacrifice qui appartient à la logique du jour et conforte sa domination.

53 Ibid., p. 275.

${ }^{54} \mathrm{EH}$, p. 162.

${ }^{5}$ Ibid., p. 170.

${ }^{56}$ LS, p. 323 : «Seule cette manière de voir est à même de nous ouvrir une nouvelle approche de l'idée de la liberté, de nous faire comprendre que la liberté est quelque chose de négatif [...] ».
} 
vue d'autre chose, qui n'est pas une étape vers..., quelque chose audelà et au-dessus de quoi il ne peut rien y avoir. Le sommet est là même, dans ce dessaisissement de soi auquel les hommes sont appelés, pour lequel ils sont arrachés à leur emploi, à leurs talents, à leurs possibilités, à leur avenir ${ }^{57}$.

Il s'ensuit de ces analyses que cette autre guerre dans la guerre modifie profondément le statut de l'ennemi. En effet, dans ce combat contre le jour et l'ordre, par conséquent contre le monde de la vie préhistorique et a-critique, l'adversaire ne se trouve pas forcément dans l'autre camp. Les uns et les autres, des deux côtés des tranchées pour ainsi dire, affrontent sans même le savoir la domination du jour. La conséquence de l'autre guerre, la guerre existentiale et aléthique, c'est que l'ennemi n'est plus " un adversaire absolu sur le chemin de la volonté de paix ${ }^{58}$. Il peut devenir un allié. Il participe à la même situation que nous, de sorte que nous devenons complices dans l'ébranlement $\mathrm{du}$ jour. L'homme n'est pas toujours seul dans le troisième mouvement de l'existence qui perce dans la guerre. Tel est le sens de ce que Patočka nomme la "solidarité des ébranlés " 59 , expression si souvent citée et mal comprise. Les ébranlés sont ceux qui font l'expérience totale et absolue du front de sorte qu'elle les amène à remettre en cause, par le sacrifice total, le sens donné de la vie et de la société en vue d'un questionnement libre et ouvert. Ils sont ébranlés "dans leur foi en le jour, la vie et la paix $»^{60}$. En tant qu'ils participent de cette critique et destruction du donné, les ébranlés sont aussi les ébranleurs. Ils forment l'ensemble de ceux qui s'exposent dans les deux camps, en apparence opposés, à l'ébranlement radical et violent du sens. Malgré leurs différends, ils appartiennent à une même révélation nocturne du problématique. Il y a donc une "entente dans l'opposition $»^{61}$. La solidarité des ébranlés, qui permet de dépasser la solitude du soldat exposé à cette déhiscence du sens, signifie dès lors cette harmonie dans le conflit se manifestant dans le combat commun contre le jour. Elle ne renvoie pas, comme on peut aisément s'en apercevoir à présent, à la compassion pour les victimes, et au droit d'ingérence (à savoir à l'exigence morale d'une solidarité internationale envers elles), mais, à rebours de cette version édulcorée, à la passion

\footnotetext{
${ }^{57} \mathrm{EH}$, p. 166. Même idée, p. 171 : «Il s'agit de comprendre que c'est ici que se joue le véritable drame de la liberté ; la liberté ne commence pas seulement après, une fois le combat terminé ; au contraire, sa place est précisément dans ce combat - voilà le punctum saliens, le sommet marquant, du haut duquel on peut embrasser du regard tout le champ de bataille ».

${ }^{58}$ Ibid., p. 167.

${ }^{59}$ Ibid., p. 67, 167, 171-172.

${ }^{60}$ Ibid., p. 172.

${ }^{61} \mathrm{Ibid} .$, p. 167.
} 
extrême de la destruction (l'ébranlement) qui saisit les combattants et les fait accéder à un au-delà de l'être: "La solidarité des ébranlés peut se permettre de dire non aux mesures de mobilisation qui éternisent l'état de guerre. Elle ne dressera pas de programmes positifs ; son langage sera celui du démon de Socrate : tout en avertissements et interdits ${ }^{62}$. C'est un front silencieux qui s'édifie dans la persécution et les incertitudes, mais qui délivre une fin de non-recevoir à toute nouvelle mobilisation de la force. Cette solidarité paradoxale qui implique à la fois l'exposition totale au danger et la compréhension du caractère totalitaire de la force, unit ceux qui sont divisés. On retrouve ici chez Patočka le motif héraclitéen de l'unité des contraires, du polemos qui sépare et joint les opposés :

À l'aube de l'histoire, Héraclite d'Éphèse formulait son idée de la guerre comme loi divine dont se nourrissent toutes les lois humaines. Il n'entendait pas la guerre au sens d'une expansion de la vie, mais comme prééminence de la Nuit, volonté d'affronter librement le péril dans l'aristeia, la preuve d'excellence à l'extrême limite des possibilités humaines que choisissent les meilleurs en se décidant à échanger le prolongement éphémère d'une vie confortable contre une célébrité durable dans la mémoire des mortels. Ce conflit est père des lois de la cité, père de toutes choses; il montre que les uns sont esclaves, les autres libres ${ }^{63}$.

Alors que la guerre du jour confronte deux camps qui pensent incarner le sens et la vie, de sorte que toute guerre est une guerre civile de la nature elle-même, si l'on peut dire, la guerre de la nuit, plus secrète et souterraine, révèle une même opposition aux certitudes données et une exposition tragique au rien. C'est seulement dans l'ébranlement assourdissant et métallique de la guerre moderne que les membres des deux camps prennent conscience, au bord du gouffre, d'un au-delà de la vie, et que l'histoire relève d'un conflit perpétuel de la vie nue et de la vie au sommet. « La solidarité des ébranlés, c'est la solidarité de ceux qui comprennent ${ }^{64}$. Et que comprennent-ils dans la situation extrême du front? Précisément que «l'histoire est ce conflit de la vie nue, enchaînée par la peur, avec la vie au sommet, qui ne planifie pas le quotidien à venir, mais voit clairement que le jour ordinaire, sa vie et sa paix auront une fin ${ }^{65}$. Si les ennemis s'opposent dans la guerre du jour, ils s'unissent dans sa contes-

\footnotetext{
${ }^{62}$ Ibid., p. 172.

${ }_{63} \mathrm{EH}, \mathrm{p} .173$.

${ }^{64} \mathrm{Ibid} .$, p. 172 . Cf. p. 173 : «ébranler le quotidien des factologues et des routiniers, leur faire comprendre que leur place est de ce côté du front, et non pas auprès des mots d'ordre du jour, si séduisants soient-ils ».

${ }^{65} \mathrm{Idem}$.
} 
tation nocturne. Ils appartiennent en fin de compte à un même camp, celui de ceux qui disent non à la reproduction immanente de la vie et qui, prenant conscience de leur historicité, recherchent un sens plus haut, plus ample, plus complexe. C'est en ce sens-là que polemos « n'est pas la passion dévastatrice d'un envahisseur sauvage mais au contraire un créateur d'unité ${ }^{66}$.

Toutefois cette expérience du front vise à mettre fin aux politiques de mobilisation «qui éternisent l'état de guerre ${ }^{67}$. Autrement dit $l^{\prime}$ autre guerre, la guerre du sens, aspire à une « paix réelle $»^{68}$ qui ne soit pas la continuation de la guerre au profit de l'ordre borné du jour. La solidarité des ébranlés constitue donc une étape fondamentale dans le refus du donné, du donné comme guerre ontique et naturelle. Elle seule peut mener à une véritable paix qui se différencie de la paix illusoire du jour, laquelle maintient le statu quo, prolonge la guerre dans la vie, le travail, les loisirs. La paix réelle ne sortira, si elle doit sortir, que de ce choc frontal, et non de la paix armée de l'ordre mondial. Grâce à une mobilisation totale du vivant, elle produit, par un ultime basculement, et de manière renversante, la démobilisation totale, l'accès au rapport libre, historique et détaché à la vie, à savoir l'existence authentique : "L'humanité n'atteindra pas le terrain de la paix en se laissant prendre aux leurres de la quotidienneté, en se mesurant à l'aune du jour ${ }^{69}$. Au contraire, c'est en s'enfonçant dans la nuit, sans chercher à se retourner ni à fuir, encore moins à retrouver le confort consolant du donné, qu'elle pourra peut-être instaurer un jour une paix qui ne soit pas l'expression de la domination de l'ordre vital :

La guerre peut faire apparaître que, parmi les hommes libres, certains sont capables de devenir des dieux, de toucher à la divinité, à ce qui constitue l'unité dernière et le mystère de l'être. Ce sont ceux qui comprennent que polemos n'a rien d'unilatéral, qu'il ne divise pas, mais unit, que les ennemis ne sont des touts distincts qu'en apparence, qu'ils sont en réalité inséparables dans l'ébranlement commun du quotidien ${ }^{70} \ldots$

Or seule la guerre, qui ne se réduit pas à l'expérience de tuer et d'être tué, peut ouvrir ce champ nouveau du dépassement de la vie, du sens et de l'être. Elle est le sacrifice total qui révèle l'excellence de l'âme. Car c'est par cette exposition brute à la mort violente que

\footnotetext{
66 Ibid., p. 67.

${ }^{67}$ Ibid., p. 172.

${ }^{68}$ Ibid., p. 170.

${ }^{69}$ Ibid., p. 173.

70 Ibid., p. 174.
} 
l'individu prend conscience de son être-libre en se défaisant de l'attachement à la vie, en accédant à une dimension supérieure de l'existence. On le voit, pour Patočka, l'existant ne conquiert son authenticité éthique et aléthique, non dans son être-pour-le-mort, mais dans son être-pour-la-guerre, dans le courage de s'exposer à la destruction violente des certitudes et de la vie, afin de laisser poindre ce qui les dépasse. Ce sacrifice, qui n'est pas sacrifice pour le salut de sa famille ou de sa cité (l'alternative de Hegel ne vaut plus ici, car elle demeure immanente à la vie et oppose deux conceptions bornées de la totalité : la vie privée et la vie publique), ce sacrifice qui n'est ni socratique ni machiavélien, est un sacrifice pour rien, un sacrifice problématique qui manifeste, contre la puissance de la vie, la puissance ascétique de critique et de détachement de la vie qui n'est autre, pour Patočka, que la liberté elle-même. 